\title{
Hábitos de compra de alimentos da população de Ouro Preto (Minas Gerais)
}

\author{
Silvana Pedroso de Oliveira ${ }^{1}$, Lidiane Batista Muniz ${ }^{2}$, Claudia Aparecida Marlière ${ }^{3}$, \\ Silvia Nascimento Freitas ${ }^{3}$, Karina Zanotti Fonseca ${ }^{2}$, Ligia Ribeiro Carvalho ${ }^{2}$, Vicente Garibay Cancho ${ }^{4}$
}

Os hábitos de compra de alimentos vão influir no padrão de consumo e na segurança alimentar das famílias. Com o objetivo de avaliar os hábitos de compra de alimentos no Município de Ouro Preto, Minas Gerais, foi aplicado um questionário a moradores de cento e trinta e sete domicílios, selecionados por amostragem sistemática. O maior gasto com alimentação ocorreu nos supermercados. Os estabelecimentos mais freqüentados foram: açougues, supermercados, padarias e quitandas. Os principais critérios para escolha dos alimentos foram preço e qualidade; para escolha do local de compra os critérios foram preço e higiene do local. A maioria dos entrevistados declarou que planejava suas compras, e mais da metade deles informou que fazia compras a crédito, principalmente nos supermercados e açougues. Os entrevistados costumavam ler as informações contidas nos rótulos dos produtos, principalmente a data de validade, a informação nutricional e a composição do produto. Os principais problemas para o abastecimento de alimentos apontados foram: alto preço dos produtos e número insuficiente de estabelecimentos comerciais no bairro. Os resultados revelam preferência pelo pequeno comércio especializado (açougues, padarias e quitandas) e também pelos supermercados. De maneira geral, observa-se que o preço foi o principal critério considerado, tanto na escolha do alimento como do estabelecimento comercial, o que pode ser associado à baixa renda dos entrevistados.

Palavras-chave: hábitos de compra, consumo alimentar, Ouro Preto

\section{Food purchase habits of the population of Ouro Preto (Minas Gerais)}

Food purchase habits influence the pattern of food consumption and security of a population. In order to evaluate the habits of food purchasing in the city of Ouro Preto, Minas Gerais, a questionnaire was applied to the residents of one hundred and thirty-seven households, selected by systematic sampling. Major food expenses occurred in supermarkets. The most frequented establishments were butcher shops, supermarkets, bakeries and greengrocery stores. The main criteria for the selection of a food were price and quality and for choosing the local of purchase, price and hygiene. The majority of the subjects interviewed declared planning their purchases and more than half informed they bought on credit, mainly at supermarkets and butcher shops. The interviewed read the label information on food products, especially the expiry date, nutritional information and food composition. The main problems identified with the food produt supply were: high prices and the insufficient number of commercial establishments in the district. The results revealed a preference for small, specialized shops (butcher shops, bakeries and greengroceries) and also for supermarkets. In general, it was observed that the price was the main criteria of choice in either kind of food or commercial establishment, which can be associated with the low income of the interviewed.

Keywords: purchase habits, food consumption, Ouro Preto

\footnotetext{
${ }^{1}$ Pesquisadora - Embrapa Agroindústria de Alimentos - Av. das Américas, 29.501 - Guaratiba - CEP 23020-470 - Rio de Janeiro - RJ

- e-mail: silvana@ctaa.embrapa.br

${ }^{2}$ Nutricionistas - bolsistas da Escola de Nutrição - Universidade Federal de Ouro Preto - Ouro Preto - MG

${ }^{3}$ Professoras da Escola de Nutrição - Universidade Federal de Ouro Preto - Ouro Preto - MG

${ }^{4}$ Professor do Instituto de Ciências Exatas e Biológicas - Universidade Federal de Ouro Preto - Ouro Preto - MG
} 


\section{Introdução}

A segurança alimentar das famílias depende, fundamentalmente, da capacidade destas obterem alimentos em quantidade suficiente, de modo a garantir uma ingestão dietética adequada e permanente a todos os seus membros, permitindo assim levarem uma vida ativa e saudável (FAO/OMS, 1992).

De acordo com HOFFMAN (1996) e VALENTE (1997), o problema da segurança alimentar no Brasil se deve, principalmente, à dificuldade de acesso ao alimento por uma grande parte da população, devido ao baixo poder aquisitivo e não à falta de alimentos.

Os hábitos de compra e de consumo de alimentos estão interligados e dependem de fatores econômicos e culturais (OLIVEIRA e THEBAUDMONY, 1998). Além disso, a falta de planejamento para a compra de alimentos pode levar a desperdícios e/ou à preferência e aquisição de produtos supérfluos, em detrimento da aquisição de alimentos necessários para a promoção e manutenção da saúde, o que compromete a segurança alimentar das famílias.

Segundo NABUCO e PORTO (2000), um registro dos locais de compra dos consumidores revelará a importância dos equipamentos públicos e privados de abastecimento presentes no Município, a tipologia destes equipamentos segundo a escala de venda, a distribuição espacial dos mesmos e o conhecimento das principais demandas do consumidor.

Geralmente, os métodos de inquérito alimentar disponíveis não levam em consideração os critérios de escolha dos alimentos pelos consumidores, os hábitos de compra dos indivíduos e a forma pela qual estes fatores poderiam influenciar no padrão de consumo e no estado nutricional da população (THOMPSON e BYERS, 1994; OLIVEIRA e THEBAUD-MONY, 1997).

Este trabalho teve como objetivo avaliar os hábitos de compra de alimentos e identificar fatores que possam influenciar o padrão de consumo e a segurança alimentar das famílias, no Município de Ouro Preto, Estado de Minas Gerais.

\section{Metodologia}

O distrito-sede de Ouro Preto é dividido em trinta e seis setores censitários. Foram sorteados, através de amostragem sistemática, cinco setores, e, para cada um deles, foram considerados $10 \%$ dos domicílios, resultando num total de 154 residências a serem pesquisadas. As informações relativas aos setores censitários e domicílios foram obtidas junto ao escritório do IBGE, situado no Município de Ouro Preto.

Foi elaborado um questionário visando avaliar, entre outros aspectos, os hábitos de compra das famílias residentes em Ouro Preto, com base no estudo multicêntrico sobre consumo alimentar (INAN-MS / NEPA, 1997) e na pesquisa realizada por OLIVEIRA e THEBAUD-MONY (1998). Foram coletadas as seguintes informações socioeconômicas para caracterização dos entrevistados: idade, escolaridade, sexo, ocupação e renda familiar mensal per capita. Os participantes da pesquisa foram questionados sobre os critérios de escolha dos alimentos e do estabelecimento comercial para a compra dos mesmos, a freqüência de compra em um determinado estabelecimento, o gasto médio com alimentação em geral e em cada tipo de estabelecimento, o planejamento realizado para as compras, a ocorrência de compra a crédito, os problemas de abastecimento de alimentos e a leitura de rótulos dos produtos alimentícios.

Foram entrevistados os responsáveis pela compra de alimentos no domicílio, em geral mulheres. Os questionários foram aplicados por entrevistadores devidamente treinados, graduandos do curso de Nutrição da Universidade Federal de Ouro Preto, no período de março a junho de 2001.

Os dados foram analisados a partir do cálculo das médias e das freqüências para as variáveis selecionadas.

\section{Resultados e Discussão}

Foram analisados 137 questionários, resultando numa perda de 11,04\% em relação à amostra inicial, justificada pela inexistência de alguns números sorteados e inconsistência dos dados coletados.

A maioria dos entrevistados era do sexo feminino 
$(89,8 \%)$, sendo que entre elas $29,9 \%$ eram chefes do domicílio. As ocupações mais comuns declaradas pelos entrevistados foram "do lar" (32,1\%), aposentados $(13,9 \%)$ e empregadas domésticas $(10,2 \%)$. A média de idade dos entrevistados foi 44 anos. Quanto ao nível de escolaridade, $16,8 \%$ tinham o primário completo (até a $4^{a}$ série do primeiro grau), $23,4 \%$ cursaram o primeiro grau e apenas $10,2 \%$ possuíam o segundo grau completo.

$\mathrm{Na}$ Tabela 1 encontram-se os dados referentes à renda familiar mensal per capita dos entrevistados. Verifica-se que a maioria deles apresentou renda baixa, $30,7 \%$ possuíam renda entre 0,5 e 1,0 SMPC (salários mínimos per capita) e 35,0\% entre 1,0 e 3,0 SMPC.

A renda média mensal per capita das famílias entrevistadas, em Ouro Preto, foi de R $\$ 298,30$, enquanto que a renda total foi de $\mathrm{R} \$ 1019,01$. A partir dos dados da FUNDAÇÃO JOÃO PINHEIRO (2004), em 2002 a renda per capita mensal estimada a partir do Produto Interno Bruto para Minas Gerais era em torno de $\mathrm{R} \$$ 530,81 e para Ouro Preto R\$939,95.

$\mathrm{Na}$ Tabela 2 são encontradas a porcentagem de entrevistados que fazem compras nos diferentes estabelecimentos comerciais de alimentos e o respectivo gasto médio mensal com alimentação. Os dados mostram que a maioria dos entrevistados faz compra de alimentos em açougues $(99,3 \%)$, supermercados $(94,9 \%)$, padarias $(86,9 \%)$ e quitandas $(73,0 \%)$, sendo estes, portanto, os estabelecimentos mais freqüentados. Verifica-se que as quitandas foram mais freqüentadas do que as feiras e os sacolões/ varejões.
Tabela 1. Distribuição percentual dos entrevistados de acordo com a renda familiar mensal per capita (número e \% de entrevistados). Ouro Preto, Minas Gerais, 2001.

\begin{tabular}{lcc}
\hline Renda familiar per capita em SM $\left(^{*}\right)$ & $\mathrm{N}$ & $\mathbf{( \% )}$ \\
\hline$\leq 0,5$ & 13 & 9,5 \\
$>0,5 \leq 1,0$ & 42 & 30,7 \\
$>1,0 \leq 3,0$ & 48 & 35,0 \\
$>3,0 \leq 5,0$ & 17 & 12,4 \\
$>5,0$ & 7 & 5,1 \\
Não informou & 10 & 7,3 \\
\hline Total & 137 & 100 \\
\hline
\end{tabular}

(*) SM =Salários mínimos; na época da entrevista, 1 salário mínimo correspondia a $\mathrm{R} \$ 151,00$.

Vários estudos têm verificado a tendência de crescimento das vendas dos supermercados, em detrimento do comércio tradicional, representado pelos armazéns e mercearias (POLETTO et al, 1996; CARMO, 1996; NEVES et al., 2000).

MALUF (2000), comparando os dados da Pesquisa de Orçamentos Familiares do IBGE, de 1987 e 1996, observou que os supermercados aparecem como o principal local de compra de alimentos, seguidos dos estabelecimentos especializados (açougues e padarias).

OLIVEIRA e THEBAUD-MONY (1998) realizaram um estudo em três localidades na cidade de São Paulo, as quais apresentavam características

Tabela 2. Distribuição percentual dos entrevistados de acordo com o local de compra de alimentos e gasto médio mensal por estabelecimento. Ouro Preto, Minas Gerais, 2001

\begin{tabular}{lcc}
\hline Tipo de Estabelecimento & Porcentagem dos entrevistados $(\mathrm{n}=137)$ & Gasto médio mensal $(\mathrm{R} \$)(\mathrm{n}=126)$ \\
\hline Supermercado & 94,9 & 171,37 \\
Açougue & 99,3 & 44,62 \\
Quitanda & 73,0 & 25,69 \\
Padaria & 86,9 & 27,13 \\
Mercearia & 49,6 & 16,63 \\
Feira & 52,6 & 11,14 \\
Varejão/sacolão & 39,4 & 10,92 \\
Armazém & 24,1 & 6,75 \\
Vendedor ambulante & 30,7 & 2,73 \\
\hline
\end{tabular}


socioeconômicas distintas. Os autores observaram que o supermercado foi o principal estabelecimento para a compra de alimentos. As feiras eram mais procuradas do que os sacolões pelo grupo de menor poder aquisitivo, pois, segundo os autores, eles podiam comprar em pequenas quantidades e gastar menos. $\mathrm{O}$ pequeno comércio era procurado pela proximidade e pela possibilidade de obtenção de crédito. Quanto ao comportamento dos entrevistados em relação à compra, os autores observaram, de uma maneira geral, a procura por promoções.

Ainda na Tabela 2, no que se refere aos gastos mensais com alimentação, observa-se que as maiores despesas ocorreram nos supermercados e nos açougues, os quais foram também os estabelecimentos mais freqüentados pelos entrevistados. Constatou-se que, embora os estabelecimentos especializados (açougues e padarias) fossem freqüentados por um grande número de entrevistados, o supermercado aparece como o principal local de compra, em função do gasto médio mensal observado. Vale ressaltar a dificuldade dos entrevistados em fornecer a informação relativa aos gastos com alimentação e por estabelecimento, sendo que neste estudo onze pessoas não responderam a essa questão.

O gasto médio mensal com alimentação das famílias foi de $R \$ 316,98$, ou seja, em torno de 2 salários mínimos da época em que as entrevistas foram realizadas, o que representa cerca de $30 \%$ da renda total familiar ( $\mathrm{R} \$ 1019,01)$.

No Estudo Multicêntrico sobre Consumo Alimentar, realizado em 1996/97, os dados obtidos para os municípios de Campinas, Goiânia e Ouro Preto revelaram que é bastante significativa a participação do gasto médio com alimentação no orçamento familiar, sendo que, no caso de Ouro Preto, para a menor faixa de renda (até 2,5 salários mínimos), o gasto com alimentação foi de 2,91 salários mínimos, ou seja, acima do rendimento mensal familiar (INANMS/NEPA, 1997).

A maioria dos entrevistados (79,5\%) afirmou que costumava planejar suas compras e mais da metade deles $(56,2 \%)$ informou que comprava a crédito. Dos que compravam a crédito, 49,4\% utilizavam essa forma de pagamento em supermercados, $27,3 \%$ em açougues e $14,3 \%$ em padarias e quitandas. Portanto, observouse um maior número de pessoas que compram alimentos a crédito nos supermercados e açougues, os quais correspondem aos estabelecimentos onde ocorreram os maiores gastos com alimentação.

OLIVEIRA et al. (2003), em estudo sobre o comércio de alimentos em Ouro Preto, observaram, dentre as principais estratégias de venda utilizadas, as promoções, o uso de caderneta e a venda unitária, ou seja, aquela que é feita em pequenas quantidades para uso imediato, caracterizando a compra a crédito e em pequenas porções. Segundo os autores, os dados obtidos demonstraram a necessidade de se melhorar a condição socioeconômica da população para facilitar o acesso aos alimentos, uma vez que a mesma foi apontada, por vários entrevistados, como uma das principais dificuldades para o comércio de alimentos em Ouro Preto.

Quanto aos alimentos comprados a crédito, destacaram-se as carnes e derivados, os alimentos que compõem a cesta básica, as hortaliças folhosas, as hortaliças frutosas, as frutas e os laticínios, mencionados por $50,7 \%, 43,3 \%, 43,3 \%, 38,8 \%$, $34,3 \%$ e $34,3 \%$ dos entrevistados, respectivamente. Isso coincide com o que foi observado anteriormente em relação aos estabelecimentos mais freqüentados, onde se gasta mais com alimentação e onde mais são adquiridos alimentos a crédito.

$\mathrm{Na}$ Tabela 3 pode-se observar que as compras no supermercado ocorrem uma vez por mês para a maioria dos entrevistados $(69,3 \%)$. Nos açougues, quitandas e feiras, a freqüência de compra é de uma vez na semana para $43,8 \%, 37,2 \%$ e $31,4 \%$ dos entrevistados, respectivamente. Nas padarias, a freqüência de compra é diária para mais da metade dos entrevistados. Portanto, as compras feitas nos estabelecimentos especializados (açougues, padarias, quitandas e feiras) são mais freqüentes do que nos supermercados, provavelmente em função do tipo de alimento comercializado.

$\mathrm{Na}$ Tabela 4 estão apresentados os critérios de escolha dos entrevistados, em relação aos alimentos. Nota-se que o preço e a qualidade foram os principais itens considerados na escolha dos alimentos. Destacam-se, também, a preocupação com a saúde, o valor nutricional e o hábito alimentar. Sabe-se que existe, atualmente, uma maior preocupação por parte dos consumidores, no que se refere à alimentação saudável (FONSECA et al., 1998; CERVEIRA e CASTRO, 1999; NEVES et al., 2000; SANTOS e BARROS FILHO, 2002). 
Hábitos de compra de alimentos, Oliveira et al.

Tabela 3. Distribuição percentual dos entrevistados de acordo com a freqüência de compra por estabelecimento comercial. Ouro Preto, Minas Gerais, $2001(\mathrm{n}=137)$

\begin{tabular}{lccccccc}
\hline Estabelecimentos & $\begin{array}{c}\text { Todos os } \\
\text { dias }\end{array}$ & $\begin{array}{c}\text { Duas a três } \\
\text { vezes na semana }\end{array}$ & $\begin{array}{c}\text { Uma vez } \\
\text { na semana }\end{array}$ & $\begin{array}{c}\text { De quinze a } \\
\text { quinze dias }\end{array}$ & $\begin{array}{c}\text { Uma vez } \\
\text { por mês }\end{array}$ & Raramente & Nunca \\
\hline Supermercado & 1,5 & 2,2 & 9,5 & 5,8 & 69,3 & 6,6 & 5,1 \\
Quitanda & 3,6 & 6,6 & 37,2 & 13,1 & 5,1 & 7,3 & 27,0 \\
Açougue & 2,2 & 13,9 & 43,8 & 13,9 & 10,9 & 6,6 & 8,8 \\
Feira & 0,7 & 1,5 & 31,4 & 6,6 & 2,9 & 9,5 & 47,4 \\
Mercearia & 0,7 & 4,4 & 7,3 & 7,3 & 13,9 & 16,1 & 50,4 \\
Padaria & 53,3 & 14,6 & 3,6 & 3,6 & 2,9 & 8,8 & 13,1 \\
Varejão/Sacolão & 1,5 & 5,1 & 16,8 & 5,1 & 2,2 & 8,8 & 60,6 \\
Vendedor ambulante & 0,7 & 3,6 & 12,4 & 1,5 & 2,2 & 10,2 & 69,3 \\
Armazém & 0,0 & 2,2 & 2,9 & 1,5 & 8,0 & 9,5 & 75,9 \\
\hline
\end{tabular}

Tabela 4. Distribuição percentual dos entrevistados de acordo com os critérios de escolha de alimentos. Ouro Preto, Minas Gerais, $2001(\mathrm{n}=136)$

\begin{tabular}{lc}
\hline Critérios para escolha dos alimentos & \% de entrevistados \\
\hline Preço & 83,9 \\
Qualidade & 73,0 \\
Bom para a saúde & 54,5 \\
Valor nutritivo & 38,7 \\
Hábito alimentar & 37,9 \\
Informação no rótulo & 34,3 \\
Aparência do produto & 32,8 \\
Alimento natural & 30,6 \\
Facilidade no preparo & 27,7 \\
Ausência de conservadores & 19,7 \\
Embalagem & 16,1 \\
\hline
\end{tabular}

Em estudo realizado por LENNERNÄS et al. (1997), sobre os critérios de escolha de alimentos por adultos da União Européia, verificou-se que qualidade, preço, sabor, tentativa de ter uma alimentação saudável e "o que minha família quer comer" estavam entre os cinco principais. Segundo estes autores, em geral as influências observadas para a escolha dos alimentos parecem estar mais relacionadas a fatores demográficos do que culturais. As mulheres e as pessoas idosas e com maior nível de escolaridade são mais propensas do que outros indivíduos a considerar tentativa de ter uma alimentação saudável na escolha dos alimentos. Qualidade, preço, tentativa de ter uma alimentação saudável e preferências familiares parecem ser mais importantes para as mulheres, enquanto que sabor e hábito alimentar são mais freqüentemente apontados pelos homens. O preço parece ser mais importante entre os desempregados e os aposentados.

Em estudo mencionado anteriormente, realizado no Município de São Paulo, OLIVEIRA e THEBAUD-MONY (1998) observaram que, para as 
camadas de menor renda, o preço apareceu em primeiro lugar como critério de escolha dos alimentos, seguido da qualidade, enquanto que nos grupos de maior renda ocorreu o contrário. A facilidade de preparo, o valor nutricional e a busca por novos produtos também foram considerados, sobretudo pelos grupos de renda média e alta.

Segundo JUNQUEIRA (1995), os brasileiros das camadas de menor renda, principalmente, passaram a consumir mais e, também, optaram pela compra de produtos de melhor qualidade, desde a implantação do Plano Real. Atualmente, sabe-se da preocupação crescente dos consumidores em relação à qualidade e à segurança dos alimentos (NEVES et al., 2000; VIEIRA e SALAY, 2001).

De acordo com a Tabela 5, entre os critérios considerados para a escolha do local de compra dos alimentos, destaca-se o preço. A higiene do local e a entrega em domicílio contribuem para a escolha do local de compra de alimentos, para cerca da metade dos entrevistados. A qualidade dos alimentos aparece em quinto lugar, sendo um motivo de preocupação, em relação à escolha dos estabelecimentos, para 41,6\% dos entrevistados.
FONSECA et al. (1998) estudaram, em Campinas, a atitude do consumidor, em relação à compra de hortifrutícolas em hipermercados e sacolões/ varejões e verificaram que, além da qualidade, a limpeza e a facilidade de localização foram os itens mais considerados para a escolha do estabelecimento.

No que se refere à leitura dos rótulos dos produtos alimentícios, cujas informações poderiam ser consideradas na escolha dos alimentos, $83,2 \%$ dos entrevistados declararam que costumam lê-las. Destes, $94,7 \%$ lêem a data de validade, $43,0 \%$ a informação nutricional e $29,8 \%$ a composição do produto.

Segundo BRANDÃO et al. (1991), a data de validade do alimento é cada vez mais questionada e controlada pelos consumidores no comércio varejista.

Estudo realizado por OLIVEIRA e MARUCCI (1998), na cidade de São Paulo, sobre o comportamento do consumidor adulto e idoso em relação à informação contida nos rótulos dos produtos alimentícios, mostrou que a maioria dos entrevistados $(93,1 \%)$ costumava lê-la, principalmente a data de

Tabela 5. Distribuição percentual dos entrevistados de acordo com os critérios de escolha do local de compra de alimentos. Ouro Preto, Minas Gerais, $2001(\mathrm{n}=136)$

\begin{tabular}{lc}
\hline Critérios para escolha do local de compra & \% de entrevistados \\
\hline Preço & 70,8 \\
Higiene do local & 52,6 \\
Entrega em domicílio & 49,6 \\
Variedade dos alimentos & 42,3 \\
Qualidade dos alimentos & 41,6 \\
Localização & 37,9 \\
Higiene dos funcionários & 34,3 \\
Promoção & 27,0 \\
Crédito & 25,5 \\
Venda unitária (em pequenas quantidades) & 18,9 \\
Propaganda & 13,1 \\
Bom atendimento & 6,5 \\
Hábito de comprar no local & 0,7 \\
\hline
\end{tabular}


validade $(99,2 \%)$, os ingredientes $(74,4 \%)$ e a informação nutricional $(50,4 \%)$.

Visando verificar qual a intenção de compra dos entrevistados, no caso de aumento da renda, perguntou-se aos mesmos o que comprariam se pudessem gastar mais com alimentação. Destacaramse, como resposta, as frutas $(26,3 \%$ dos entrevistados), as carnes e derivados (17,5\%), as hortaliças folhosas $(15,3 \%)$ e os laticínios $(14,6 \%)$, sendo que $27,7 \%$ deles disseram que não comprariam mais nada. Esses dados refletem a dificuldade de acesso a alimentos que apresentam preços mais elevados.

Segundo MALUF (1999), as estruturas de abastecimento também fazem parte dos elementos que determinam as condições em que a população acessa os alimentos, ou seja, além de dispor de renda monetária regular, esta terá que se materializar numa cesta de consumo cujo custo, composição e qualidade dos bens que a integram, são definidos por agentes econômicos.

Os principais problemas de abastecimento em Ouro Preto, apontados pelos entrevistados, foram o alto preço dos alimentos (59,3\%), o número insuficiente de estabelecimentos no bairro (39,6\%), a pequena variedade dos produtos $(29,7 \%)$, a distância dos estabelecimentos em relação ao domicílio $(18,2 \%)$ e a má qualidade dos alimentos $(17,5 \%)$. Destaca-se que 33,6\% declararam que não há problemas de abastecimento no Município. Novamente, verifica-se o preço dos alimentos como um fator relevante para o abastecimento no Município, segundo os entrevistados.

Em outro estudo, realizado em Ouro Preto, para se avaliar as características do comércio de alimentos, concluiu-se, com base nas estratégias de venda e nas dificuldades citadas pelos entrevistados, que a situação econômica da população parece ser o principal obstáculo tanto para o comércio como para a segurança alimentar das famílias (OLIVEIRA et al., 2003).

UCHIMURA e BOSI (2003) analisaram as modalidades de intervenção, destacando as ações direcionadas a facilitar o acesso aos alimentos para a população de baixa renda, a partir de iniciativas de comercialização de produtos a preços mais baratos, em comparação com aqueles praticados na rede convencional de comercialização. Os autores avaliaram, mais especificamente, os programas Mercadão Popular e Armazém da Família, implantados em Curitiba, estado do Paraná, e concluíram que os mesmos procuravam cumprir a obrigação do Estado de atender aos direitos da população, de acesso à alimentação adequada. No entanto, os autores ressaltaram a necessidade de se considerar as manifestações individuais inerentes aos processos de utilização dos programas, na análise deste tipo de intervenção.

CUNHA et al. (2000) discutiram os programas da SMAB (Secretaria Municipal de Abastecimento) de Belo Horizonte, Minas Gerais, destacando os programas Restaurante Popular, Cestão Popular, Abastecer e Comboio.

Portanto, verifica-se que existem várias ações, em nível municipal, visando promover a segurança alimentar das famílias, especialmente as de baixa renda.

\section{Conclusões}

Os resultados desta pesquisa revelam que os estabelecimentos mais freqüentados para compra de alimentos, pelas famílias entrevistadas em Ouro Preto, são os supermercados, os açougues, as padarias e as quitandas, em detrimento das mercearias, feiras, sacolões e armazéns. O supermercado aparece como o mais citado local de compra de alimentos, destacando-se, também, quando é considerado o volume de gastos com alimentação.

De maneira geral, observou-se que o preço foi o principal critério considerado para a escolha dos alimentos e dos estabelecimentos, o que pode estar associado à baixa renda da população. Além disso, o preço elevado foi apontado, por mais da metade dos entrevistados, como um dos problemas do abastecimento alimentar em Ouro Preto.

Portanto, os resultados indicam a necessidade de se criar condições que possibilitem um melhor acesso aos alimentos pela população de Ouro Preto, por exemplo, a partir da implementação de programas de geração de emprego e renda ou de ações que visem à comercialização de alimentos a preços mais baixos, garantindo-se, assim, a segurança alimentar das famílias. 


\section{Agradecimentos}

Agradecemos à PROPP/UFOP e à FAPEMIG (processo CDS-2473/98) pelo apoio financeiro e à Secretaria Municipal da Fazenda, à Secretaria Municipal de Agropecuária e Meio Ambiente, à Emater/MG e ao Setor de Vigilância Sanitária da Secretaria Municipal de Saúde de Ouro Preto e também aos alunos da Escola de Nutrição da Universidade Federal de Ouro Preto pela colaboração.

\section{Referências Bibliográficas}

BRANDÃO, A.C.B.H.; BRANDÃO, A.A.H.; GERMANO, M.I.S.; GERMANO, P.M.L. Segurança alimentar nos estabelecimentos de consumo. Higiene Alimentar, São Paulo, v.5, n.19, p.20-21, 1991.

CARMO, M. S. (Re)estruturação do Sistema Agroalimentar no Brasil: a diversificação da demanda e a flexibilidade da oferta, São Paulo: IEA, 1996, 256p.

CERVEIRA, R.; CASTRO, M.C. Consumidores de produtos orgânicos da cidade de São Paulo: características de um padrão de consumo. Informações Econômicas, São Paulo, v.29, n.12, p.7-20, 1999.

CUNHA, A.R.A.A.; LEMOS, M.B.; NABUCO, M.R.; PESSOA, M.J. Políticas locais de acesso alimentar: entre a regulação e as políticas compensatórias. In: BELIK, W.; MALUF, R.S. (org.) Abastecimento e Segurança Alimentar: os limites da liberalização. Campinas, SP: IE/UNICAMP, 2000. p. 183-208.

FAO/OMS. Conferencia Internacional sobre Nutrición: Nutrición y desarollo - una evaluación mundial, Roma: FAO/OMS, 1992. 133p.

FONSECA, M.C.P.; SILVA, M.A.A.P; SALAY, E. Atitudes dos consumidores com relação à compra de hortifrutícolas em hipermercados e sacolões/ varejões na cidade de Campinas-SP. Cadernos de Debate, Campinas, v.VI, p.71-81, 1998.

FUNDAÇÃO JOÃO PINHEIRO. Produto interno bruto Minas Gerais Municípios e Regiões -1999-
2002 - Novas estimativas - Resultados preliminares, Belo Horizonte, dez 2004, disponível em: <http://www.fjp.mg.gov.br/produtos/cei/ informativo_pib_99_02.pdf $>$. Acesso em: 24 maio 2005.

HOFFMANN, R. Pobreza, insegurança alimentar e desnutrição no Brasil. In: GALEAZZI, M.A.M. (org.). Segurança Alimentar e Cidadania: A contribuição das Universidades Paulistas. Campinas: Mercado das Letras, 1996. p. 195-213.

INAN-MS/NEPA. Estudo Multicêntrico sobre Consumo Alimentar. Cadernos de Debate, Campinas, Vol. Especial, 1997.

JUNQUEIRA, A.H. O Plano Real e os seus reflexos sobre a produção e o consumo de alimentos no Brasil. Conjuntura Alimentos, São Paulo, v.7, n.1, p.4-10, 1995.

LENNERNÄS, M., FJELLSTRÖM, C., BECKER, W., GIACHETTI, I., SCHIMITT, A., REAMAUT DE WINTER, A.M., KEARNEY, M. Influences on food choice perceived to be important by nationallyrepresentative samples of adults in the European Union, European Journal of Clinical Nutrition, London, n.51, suppl. 2, p.S8-S15, 1997.

MALUF, R.S. Ações públicas locais de abastecimento, São Paulo: Pólis, 1999. 88p.

MALUF, R.S. Consumo de alimentos no Brasil: traços gerais e ações públicas locais de Segurança Alimentar, São Paulo: Pólis, 2000. 59p.

NABUCO, M.R.; PORTO, S.I. Como planejar e executar o abastecimento alimentar municipal. In: BELIK, W.; MALUF, R.S. (org.) Abastecimento e Segurança Alimentar: os limites da liberalização. Campinas, SP: IE/UNICAMP, 2000. p. 209-234.

NEVES, M.F.; CHADDAD, F.R.; LAZZARINI, S.G. Alimentos: novos tempos e conceitos na gestão de negócios, São Paulo: Editora Guazzelli Ltda, 2000, $129 \mathrm{p}$.

OLIVEIRA, S.P.; MARUCCI, M.F.N. Comportamento de consumidores adultos e idosos em relação à informação contida nos rótulos dos produtos alimentícios. In: Congresso Brasileiro de 
Ciência e Tecnologia de Alimentos, 15., 1996, Poços de Caldas. Anais do XV Congresso Brasileiro de Ciência e Tecnologia de Alimentos, v.1, p.597600, 1997.

OLIVEIRA, S.P.; THEBAUD-MONY, A. Estudo do consumo alimentar: em busca de uma abordagem multidisciplinar. Revista de Saúde Pública, São Paulo, v.31. n.2, p.201-208, 1997.

OLIVEIRA, S.P.; THEBAUD-MONY, A. Hábitos e práticas alimentares em três localidades da cidade de São Paulo. Revista de Nutrição da PUCCAMP, Campinas, v.11, n.1, p.37-50, 1998.

OLIVEIRA, S.P.; MUNIZ, L.B.; MARLIERE, C.A.; FREITAS, S.N.; CANCHO, V.G.; FREITAS, F.V.; FONSECA, K.Z.; CARVALHO, L.R. Características do comércio de alimentos no Município de Ouro Preto (Minas Gerais): subsídios para a segurança alimentar, Cadernos de Debate, Campinas, v. X, p.1-27, 2003.

POLETTO, A.C.P.; CARVALHO, J.F.; SALAY, E. Destino dos produtos hortifrutícolas comercializados na CEASA/Campinas. Revista de Política Agrícola, Brasília, Ano V, n.4, p.12-15, 1996.
SANTOS, K.M.O.; BARROS FILHO, A.A. Fontes de informação sobre nutrição e saúde utilizadas por estudantes de uma universidade privada de São Paulo. Revista de Nutrição, Campinas, v.15, n.2, p.201210, 2002.

THOMPSON, F.E.; BYERS, T. Dietary assessment resource manual. Journal of Nutrition, Philadelphia, v.124, p.2245S-2317S, 1994.

UCHIMIRA, K.Y.; BOSI, M.L.M. Programas de comercialização de alimentos: uma análise das modalidades de intervenção em interface com a cidadania. Revista de Nutrição, Campinas, v.16, n.4, p.387-397, 2003.

VALENTE, F.L.S. Do combate à fome à segurança alimentar e nutricional: o direito à alimentação adequada. Revista de Nutrição da PUCCAMP, Campinas, v.10, n.1, p.20-39, 1997.

VIEIRA, C.M.; SALAY, E. Atuação pública municipal de proteção dos consumidores frente à questão da segurança dos alimentos: o caso do PROCON- Campinas. Cadernos de Debate, Campinas, v.8, p.15-27, 2001. 